\section{Performance of rats in a runway under three concurrent conditions of reinforcement*}

\author{
MICHAEL E. RASHOTTE, Florida State Unjversity, Tallahassee, Fla. 32306 \\ and
}

ABRAM AMSEL, University of Texas at Austin, Austin, Tex. 78712

Rats can learn to run fast, fast, and slow, respectively, in discriminably different runways signaling continuous, partial, and discontinuous negatively correlated reinforcement. Performance of animals operating under such multiple reinforcement conditions in runways is compared with yoked controls, and implications for the study of transfer effects in extinction are discussed.

Discrimination learning in a runway (or differential conditioning, as it is sometimes called) usually involves two reinforcement conditions, each signaled by a discriminative stimulus, often simply $\mathrm{S} 1+$ and $\mathrm{S} 2-$. Other $t$ wo-condition-of-reinforcement discriminations (or two-component multiple schedules, in the language of operant conditioning) have been studied in the discrete-trial runway situation: continuous reinforcement (CRF) to $\mathrm{S} 1$ and partial reinforcement (PRF) to S2 (e.g., Amsel, MacKinnon, Rashotte, \& Surridge, 1964), as well as conditions involving differential magnitudes, delays, and percentages of reinforcement to the two stimuli (e.g., Henderson, 1966; MacKinnon, 1967; Spear, 1964; Spear \& Pavlik, 1966)

Recently, we have reported on rats' performance in a discrimination in which one stimulus signaled CRF while the other signaled discontinuous negatively correlated reinforcement (DNC) (Rashotte \& Amsel, 1967, 1968). On a DNC schedule, reinforcement is delivered only when $S$ runs more slowly than an arbitrary cutoff speed (Logan, 1960), and we found that during the DNC component of CRF-DNC training rats ran below this speed on about $30 \%$ of the trials. Furthermore, we reported that idiosyncratic response patterns developed in the stimulus alley related to DNC and transferred to the CRF alley in extinction.

The present paper reports the outcome of an experiment in which rats were trained concurrently in three alleys, each signaling a different reinforcement condition, $\mathrm{CRF}, \mathrm{DNC}$,

\footnotetext{
*This research was conducted at the University of Toronto and was supported by Research Grants GB-3772 from the National Science Foundation and APA-72 from the National Research Council of Canada. The authors wish to thank Michael Ross who helped collect a part of the data. Reprints may be obtained from M.E. Rashotte. Florida State University, Tallahassee, Florida 32306.
}

and PRF. We regarded the present experiment as a first step towards expanding our understanding of the transfer effects we observed in the earljer experiments. Specifically, we wanted to see if the same animal could learn different responses under two conditions of intermittent reinforcement (a slow response in the DNC alley, a fast response in the PRF) so that we might observe if and how these responses transfer to the CRF alley in extinction. yoked PRF-PRF-CRF.
SUBJECTS

The Ss were eight male albino rats of the Wistar strain, obtained from Woodlyn Farms, Guelph, Ontario. They were about 110 days old at the beginning of training.

APPARATUS

The apparatus, consisting of three runways, $63 \times 3 \times 3$ in., covered with clear Plexiglas, has been described in detail earlier (Rashotte \& Amsel, 1968). For the present experiment, one runway, $B B$, was painted flat black throughout and the other two runways were painted as "split alleys" (Kolesnik \& Amsel, 1966). That is, the left side of one of these, BW, was painted flat black and the right side flat white; in the other runway, WB, the positions of black and white were reversed. Each runway was entered from a common start segment, $13 \times 3 \times 3$ in., which narrowed to 2 in. at the exit to the runway. The start segment was painted gray and could be aligned with each runway. A microswitch attached to the start door and five photocells, at 1 - $\mathrm{ft}$ intervals along the runways, controlled .01-sec clocks which yielded five running-time measures on each trial. On reinforced trials, a Gerbrands pellet dispenser automatically dropped a 250-mg

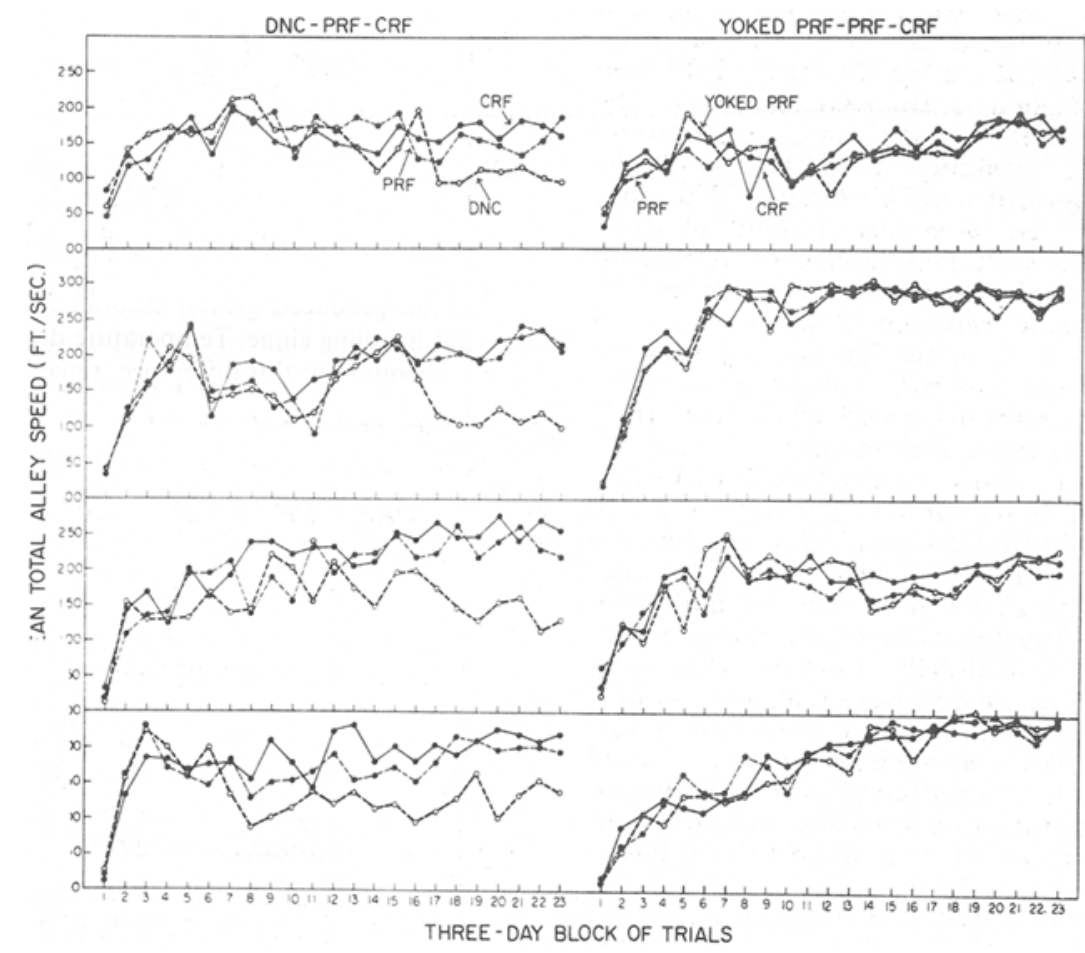

Fig. 1. Mean speed (feet/second) for individual Ss under multiple conditions of reinforcement. Each point is the mean of six speeds in each component over 3 successive days. The left column of curves is for Ss 1-4 (from top to bottom panel) trained on DNC-PRF-CRF; the right-hand column is for Ss $5-8$ trained on 
Noyes pellet into a recessed foodcup on the end wall of the appropriate runway when the rat broke the last photobeam, 3 in. from the end wall. On nonrewarded trials, the pellet dispenser did not operate. The Ss were not handled at the start of each trial, but were kept in individual carrying boxes mated to the start segment to initiate a trial. These boxes were $9 \times 3 \times 3$ in. (i.d.) and were made of unpainted plywood (see Amsel \& Rashotte, 1969).

\section{PROCEDURE}

The Ss were housed in individual cages with water freely available. Three weeks before the beginning of training, they were placed on a $24-\mathrm{h} / 10 \mathrm{~g}$ food deprivation schedule, which was maintained throughout the experiment. During this 3-week period, Ss were handled for a few minutes each day and were fed their daily ration in the home cage shortly thereafter.

Four of the Ss were assigned to run in the three stimulus alleys under the following conditions: DNC-the S found food reward only if more than $5 \mathrm{sec}$ had elapsed between opening of the start door and breaking of the last photobeam; PRF-the S found food on one trial, selected randomly, of the two such trials on each day; and CRF-reward on both of the daily trials in this alley. The DNC, PRF, and CRF components were signaled by the $\mathrm{BW}, \mathrm{WB}$, and $\mathrm{BB}$ alleys, respectively. $T h$ is training condition will be designated DNC-PRF-CRF. The four other Ss were also trained in three alleys with PRF and CRF related to two of them, but a yoked-PRF schedule replaced DNC in the third alley, i.e., in the BW runway these Ss received reward uncorrelated with their response speed when their DNC mates ran slowly enough to "earn" it. $\mathrm{Ss}$ in this yoked PRF-PRF-CRF condition were also yoked to $S$ s in the DNC-PRF-CRF condition so that the members of each pair received identical sequences of stimuli and reinforcements over all components. The yoked-PRF procedure has been used to demonstrate that slow running on a DNC schedule is related to the negative correlation between speed and the presentation of reinforcement, and not simply to the percentage or sequence of reinforcements obtained (Logan, 1960).

The order of presentation of runway stimuli within a daily session was different for each $S$ and was determined in accordance with the following restrictions: (1) In successive three-session blocks, each stimulus was first on one occasion; and (2) within each session, all three runway stimuli were encountered once before any one was presented for the second time.
With the exception of Days 1 and 2, when three trials were run, there were six trials per day, two in each runway. The intertrial interval was $10 \mathrm{~min}$.

The primary data presented in this paper are for the 414 acquisition trials (138 in each of the stimulus alleys) which were given prior to extinction training. There were also 36 extinction trials given at six trials per day. In extinction, only one DNC-PRF-CRF S and its yoked mate continued to run, unrewarded, in all three alleys. One each of the remaining three pairs was extinguished only in the CRF, or the $\mathrm{PRF}$, or the DNC/yoked-PRF alleys. Because of the small number of $S$ s and the brevity of the extinction phase, these extinction data were not very revealing, and our data concern mainly the acquisition phase.

\section{RESULTS AND DISCUSSION}

The time measures recorded on each trial were summed to yield total time from the start door to the photobeam in front of the foodcup. These time scores converted to average speeds taken over the six trials in each alley in each 3-day block. Figure 1 shows these speed scores for each of the eight Ss over the entire acquisition period.

By late acquisition, the performance of all Ss trained DNC-PRF-CRF had stabilized in the three alley components and was similar to performance under comparable two-component conditions (e.g., Rashotte \& Amsel, 1968 , Experiment 1) or performance under comparable single-schedule conditions (e.g., Rashotte \& Amsel, 1968, Experiment 2, Phase 1). That is, terminal speeds were higher in the CRF and PRF alleys than in the DNC alley. The Ss trained under yoked PRF-PRF-CRF conditions did not show systematic differences in performance in the three reinforcement conditions. Over the entire acquisition period, Ss 1.4 (those in the DNC-PRF-CRF condition) ran slowly enough to be rewarded under the DNC conditions on $24.6 \%, 23.9 \%$, $22.5 \%$, and $26.8 \%$ of trials, respectively. These figures are only slightly lower than comparable ones from our earlier experiments.

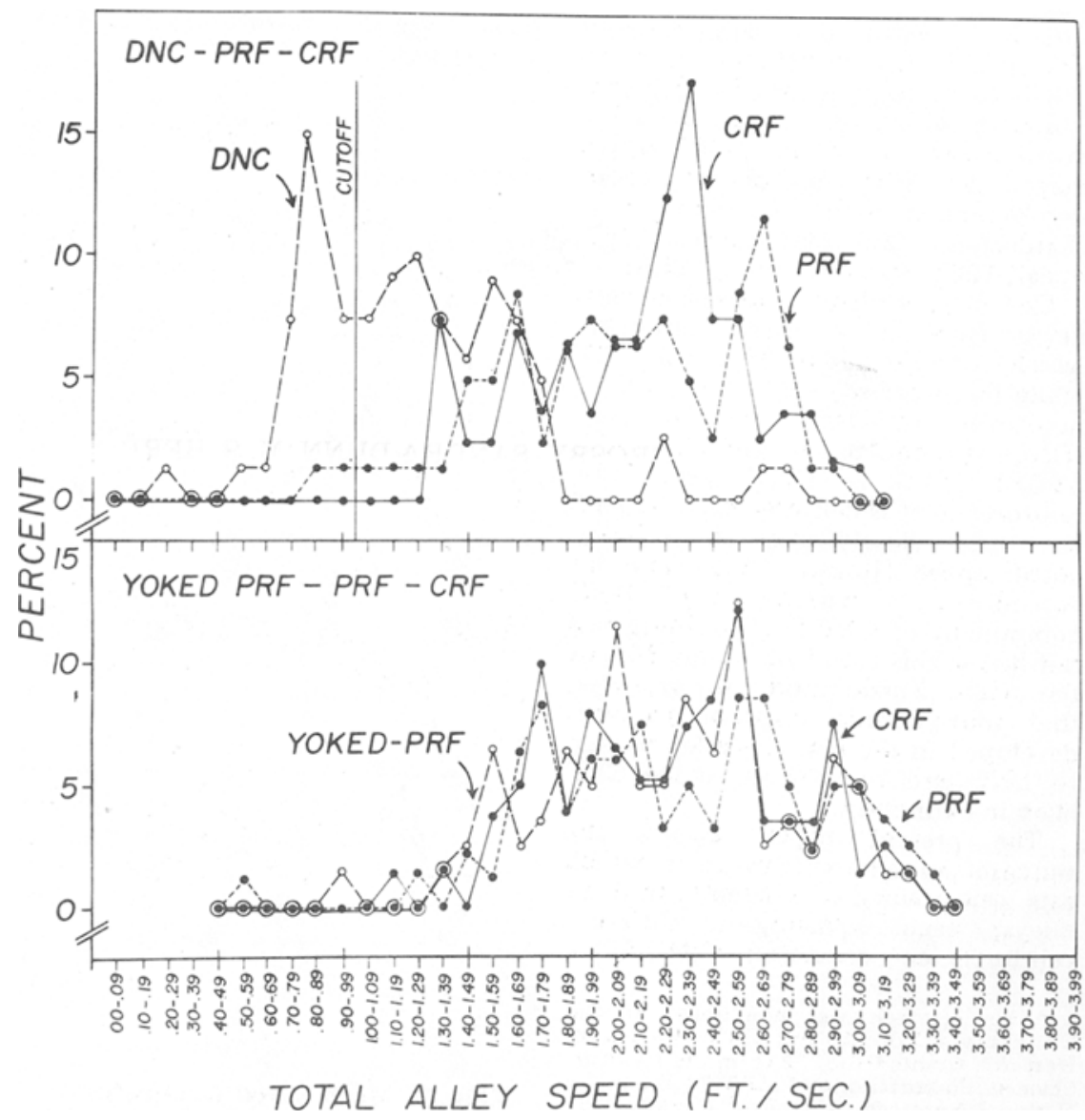

Fig. 2. Distributions of individual trial speeds in the three conditions of reinforcement of the DNC-PRF-CRF schedule (top panel), and the yoked PRF-PRF-CRF schedule (bottom panel) over the last 10 days of acquisition. These distributions are for speeds for the four Ss from each group. 
Figure 2 shows the distribution of all total alley speeds under the three reinforcement conditions in .01-sec class intervals for all four $\mathrm{Ss}$ in the DNC-PRF-CRF condition and for the yoked PRF-PRF-CRF condition. The cutoff speed below which reinforcement was presented ( $1 \mathrm{ft} / \mathrm{sec}$ ) is shown by the vertical line in the top panel. Clearly, in the DNC-PRF-CRF condition, the distribution of DNC speeds falls to the left of the PRF and CRF distributions, and an insignificant percentage of speeds in any reinforcement condition overlaps DNC in the two groups. The distributions for PRF-PRF-CRF are virtually identical. These data provide further support from within-S experiments for Logan's (1960) contention that low speed in DNC training is not simply a function of low frequencies of reinforcement, but reflects adjustment to the negatively correlated conditions of reinforcement.

A breakdown of the total alley speed plotted in Fig. 1 into speeds in the five separate runway segments in each alley showed that the low total alley speeds late in acquisition in the DNC component were due almost entirely to very low speeds in the start segment. For all Ss, these low first-segment speeds contrasted with relatively high, and virtually identical, speeds in the start measures in the PRF and CRF components. The four other runway measures were highly similar in the three reinforcement conditions. In two Ss, distinct "rituals" developed in the DNC component in the start segment: S 1 bit at a small opening in the floor between the start segment and the runway, and S 2 ran back into the start chamber after inspecting the runway stimulus. These behaviors were not observed in the start segment of the CRF or PRF alleys. Idiosyncratic behaviors of this sort were observed regularly in our earlier experiments (Amsel and Rashotte, 1969; Rashotte \& Amsel, 1968).

In our earlier experiments, we found that responses learned under DNC conditions in DNC-CRF acquisition emerged in the extinction of responding in the CRF alley (e.g., Rashotte \& Amsel, 1968), and we argued that the transfer of DNC acquisition performance to CRF extinction might be due, at least in part, to the same factors that are responsible for PRF-like extinction in the CRF alley after within-S CRF-PRF acquisition (e.g., Amsel, Rashotte, \& MacKinnon, 1966). That is, just as the slow responding of $\mathrm{DNC}$ acquisition emerges in the CRF alley during extinction, so the persistent fast responding learned in the $\mathrm{PRF}$ alley

emerges to maintain a high level of responding in the $C R F$ alley during extinction.

Now that we know that Ss can learn a slow response in a DNC alley and fast ones in PRF and CRF alleys under DNC-PRF-CRF conditions, we would of course like to know, in line with the above reasoning, whether either or both of the intermittently reinforced responses transfer to the CRF alley in extinction. Or, what are the conditions under which DNC or PRF responding is dominant over the other? The present experiment has shown that it is possible to train rats simultaneously under these reinforcement conditions in the runway, but the pilot-study nature of the extinction phase in the present experiment was not adequate to answer the above questions under experimental conditions similar to those in our earlier experiments. The PRF and CRF responses have nearly identical response topographies so that it is difficult to determine if the persistent PRF response transfers to the CRF extinction without a control group run in all three alleys under CRF. To answer questions about extinction, we will need much larger numbers of $S s$ and the CRF control condition.
AMSEL, A MACKINNON, J. R. RASHOTTE, M. E., \& SURRIDGE, C.T. Partial reinforcement (acquisition) effects within subjects. Journal of the Experimental Analysis of Behavior, 1964, 7. 135-138.

\title{
Response patterns as a factor in choice
}

\author{
PETER KILLEEN* \\ Arizona State University, Tempe, Ariz. 85281
}

In the first experiment, pigeons were given a choice between two fixed-interval 40-sec schedules. Varying the pause length by introducing stimulus changes at various times in the intervals had no effect on choice behavior. In the second experiment, pigeons were given a choice between two delays of reinforcement, and preference for the shorter delay was found to be more extreme than predicted by the matching relation. Taken together, the experiments show that preference for a schedule is more dependent on the immediacy of reinforcement than on the presence or nature of responding during the schedule chosen, and that in fixed delays of reinforcement, just as in fixed intervals, preference is more extreme than predicted from matching.

Recent studies of preference for schedules of reinforcement have shown that the major determinant of an animal's choice is how long it will have to wait to get the programmed reinforcement. The response rate engendered by the schedule chosen has scant effect on preference (Killeen, 1968). 1 The presence of stimuli

* This research was conducted with the support of NSF Grants GB 3121 and GB 3723 while I was an NIMH Predoctoral Fellow at Harvard University. Reprints are available from the Department of Psychology, Arizona State University, Tempe, Arizona 85281 . correlated with the schedule in effect is also of little importance. Neuringer (1969) pitted delays of reinforcement against equal fixed-interval (FI) schedules and found about $55 \%$ preference for the FI schedule over a large range. When a stimulus other than blackout was used to signal the delay, he found indifference between equal-valued fixed intervals and fixed delays.

For many schedules the control exerted by delay of reinforcement (or, more conveniently, immediacy of reinforcement, which is the reciprocal of the delay) is beautifully simple; 